\title{
Differential Importance Measure for Components Subjected to Aging Phenomena
}

\author{
Stefano La Rovere, ${ }^{1}$ Paolo Vestrucci, ${ }^{2}$ Maria Sperandii, ${ }^{2}$ and Claudia Mandurino ${ }^{2}$ \\ ${ }^{1}$ NIER Ingegneria, Via Altabella 3, 40127 Bologna, Italy \\ ${ }^{2}$ DIENCA, University of Bologna, Viale Risorgimento 2, 40136 Bologna, Italy \\ Correspondence should be addressed to Stefano La Rovere; s.larovere@niering.it
}

Received 27 April 2013; Revised 2 August 2013; Accepted 19 August 2013

Academic Editor: Benoit Iung

Copyright (C) 2013 Stefano La Rovere et al. This is an open access article distributed under the Creative Commons Attribution License, which permits unrestricted use, distribution, and reproduction in any medium, provided the original work is properly cited.

\begin{abstract}
The paper refers to the evaluation of the unavailability of systems made by repairable binary independent components subjected to aging phenomena. Exponential, exponential-linear, and Weibull distributions are assumed for the components failure times. We assume that components failure rate increases only slightly during the maintenance period, but we recognize the effectiveness of preventive maintenance only in presence of aging phenomena. Importance measures allow the ranking of the input variables. We propose analytical equations that allow the estimation of the first-order Differential Importance Measure (DIM) on the basis of the Birnbaum measures of components, under the hypothesis of uniform percentage changes of parameters. Without further information than that used for the estimation of "DIM for components," "DIM for parameters" allows considering separately the importance of random failures, aging phenomena, and preventive and corrective maintenance. A two-step process is proposed for the system improvement, by increasing the components reliability and maintainability performance as much as possible (within the applicable technological limits) and then by optimizing preventive maintenance on them. Some examples taken from the scientific literature are solved in order to verify the correctness of the analytical equations and to show their use.
\end{abstract}

\section{Introduction}

Several studies have demonstrated that components do not contribute to system performance in the same way [1,2]. Thus, it is essential for analysts to identify "critical" components [3]. Importance measures allow the ranking of input variables of a model [4]. This turns out to be a crucial problem when the model of the system (in a general sense) includes a variety of input variables and structural parameters. This is certainly the case of nuclear power plants and their probabilistic risk assessment [1,5-11].

In this paper, we are interested in the unavailability of systems made by repairable binary components, under "perfect," corrective, and preventive maintenance (i.e., components are "as good as new" after maintenance). We assume the exponential distribution for the components repair times.

Aging phenomena are introduced into the model through time-dependent failure rates, by assuming the Exponentiallinear distribution [5] and the more general Weibull distribution for the components failure times [7]. As in paper [8], we suppose that components failure rate increases only slightly during the "Maintenance period". It allows referring to constant "effective" failure rate of components. Differently from paper [8], but we recognize explicitly that preventive maintenance is effective only on components subjected to aging phenomena.

Importance measures allow the ranking of the input variables of the model which can be the components unavailability and/or the parameters that define their failure and repair probability distributions, according to the contribution of their changes to the model output (system unavailability). We adopt the first-order Differential Importance Measure (DIM), which provides the fraction of total change in the model output that is due to "small" one at time changes in the input variables [9].

The estimation of the "DIM for components" only requires the knowledge of the first-order partial derivatives of the system unavailability with respect to the components 
unavailability. They can be estimated on the basis of the "system function" that defines the status of the system on the basis of the state of components.

The estimation of the "DIM for parameters" provides information about the importance of the random failure, aging phenomena, and corrective and preventive maintenance separately but requires the knowledge of the first-order partial derivatives of the system unavailability with respect to each parameter. The number of partial derivatives increases as well as their complexity because of nonlinear terms.

The paper proposes analytical equations for the estimation of the first-order Differential Importance Measure (DIM) for parameters, on the basis of the same information used for the estimation of DIM for components (i.e., Birnbaum measures). Specifically, proposed equations can be applied to systems made by "independent" components. Independence among components (and among parameters that define their failure and repair probability distributions) allows considering separately the dependence of system Unavailability on components Unavailability and the dependence of each component Unavailability on its parameters. If this assumption is not fulfilled (i.e., in presence of intercomponent or functional dependences, e.g., cold spare, share load,...), and the system is described by a homogenous Markov process (this is not the case of components subjected to aging), DIM for Markov models can be used [5, 11].

In the second paragraph, we provide some general information about systems made by repairable binary components under aging phenomena and preventive maintenance $[5,7,8]$ and about the Differential Importance Measures [9]. In the third paragraph, we propose the analytical equations that allow the estimation of DIM for independent components and parameters, under different assumptions for the components failure times (exponential, exponential-linear, and Weibull). Furthermore, a two-step process is proposed for the improvement of system performance (i.e., the decrease of the system unavailability). In the fourth paragraph, we solve some examples taken from paper [8], verify the correctness of the analytical equations, and showing advantages of their use.

\section{Background}

2.1. Repairable Components with Aging Phenomena and Preventive Maintenance. We assume an exponential distribution for the failure and repair times of a binary repairable component. $\lambda$ is the (constant) failure rate and $\mu$ is the (constant) repair rate. The asymptotic value of the component unavailability is the following:

$$
U=\frac{\lambda}{\lambda+\mu} .
$$

2.1.1. Exponential-Linear Distribution. Components are assumed to age according to an exponential-linear distribution for the failure times $[5,8]$. Components failure rate increases in time according to $\lambda(t)=\lambda_{0}+a \cdot t$, where the constant term $\lambda_{0}$ refers to random failures and the "growth parameter" $a>0$ introduces aging phenomena.
Components aging is mitigated by preventive maintenance actions. We assume that the time interval between two consecutive maintenance actions, which is named maintenance period $(\tau)$, is constant during the system life time. Moreover, preventive maintenance actions are performed at regular time interval, in spite of occurrences of failures requiring corrective maintenance, and their duration is negligible.

If the components failure rate increases only slightly during the maintenance period, we can refer to a constant "Effective value"; it is estimated by imposing that the failure probabilities for the exponential-linear and the exponential distributions coincide within the following maintenance period:

$$
\lambda^{*}=\lambda_{0}+\frac{a \cdot \tau}{2}
$$

The component unavailability comes from (1) by assuming (2) for its failure rate.

2.1.2. Weibull Distribution. Components are assumed to age according to a Weibull distribution for the failure times [7]. Components failure rate increases in time according to $\lambda(t)=$ $\lambda_{0}+\beta \cdot \gamma^{\beta} \cdot t^{\beta-1}$, where the constant term $\lambda_{0}$ refers to random failures and the two parameters of the Weibull distribution introduce aging phenomena $(\beta>1$ is the shape parameter and $1 / \gamma$ is the scale parameter). If the components failure rate increases only slightly during the maintenance period, the effective value of the component failure rate turns out to be the following:

$$
\lambda^{*}=\lambda_{0}+\gamma^{\beta} \cdot \tau^{\beta-1}, \quad \text { with } \beta>1
$$

The component unavailability comes from (1), by assuming (3) for its failure rate. For $\beta=2$ and $\gamma=\sqrt{a / 2}$, the Weibull and exponential-linear distributions coincide.

2.2. Importance Measures. The unavailability of a system made by binary repairable components $U\left(\ldots, U_{i}, \ldots\right)$ comes from the system function $\phi\left(\ldots, \phi_{i}, \ldots\right)$, which defines the status of the system $(\phi=1$ if the system does not execute its "mission", $\phi=0$ elsewhere), on the basis of the state of components $\left(\phi_{i}=1\right.$ if the component " $i$ " failed, $\phi_{i}=0$ elsewhere).

Importance measures were originally introduced by Birnbaum [4]. For a system made by repairable components, the Birnbaum measure is the first-order partial derivative of the system unavailability with respect to the following component unavailability:

$$
B_{i}=\frac{\partial U}{\partial U_{i}}
$$

"Traditional" importance measures (Birnbaum measure, Criticality measure, Risk Reduction Worth, Risk Achievement Worth measures, and Fussell-Vesely) require new evaluations of the model in order to estimate the importance of a combination or group of components/parameters [7]. 
For instance, the analyst could be interested in importance of different failure modes of a component, of components with homogeneous characteristics (e.g., of the same typology), of parameters impacting different components (e.g., the inspection/maintenance frequency). Differential Importance Measure overcomes this limit [7].

DIM can be referred to the change of the components unavailability or to the change of the parameters that specify their failure and repair probability distributions. In the first case, DIM for the component " $i$ " is the fraction of the total change of the system unavailability, that is, due to the change of components unavailability, taken "one at a time" as follows:

$$
\operatorname{DIM}_{i}=\frac{\left(\partial U / \partial U_{i}\right) \cdot d U_{i}}{\sum_{j=1}^{n}\left(\partial U / \partial U_{j}\right) \cdot d U_{j}}=\frac{B_{i} \cdot \Delta_{i}}{\sum_{j=1}^{n} B_{j} \cdot \Delta_{j}},
$$

where $\Delta_{j}=1$ for the uniform changes of components unavailability $\left(d U_{i}=d U_{j}\right.$ for all $\left.i, j\right) ; \Delta_{j}=U_{j}$ for the uniform percentage changes of the components unavailability $\left(d U_{i} / U_{i}=d U_{j} / U_{j}\right.$, for all $\left.i, j\right)$.

Generally, DIM assumes different values and provides a different ranking of components under the two hypotheses; the uniform percentage changes are the more realistic ones (indeed, failure and repair rates of components differ significantly). Moreover, DIM is an additive measure, DIM for a group of components is the sum of DIM for components $\left(\mathrm{DIM}_{i, j}=\mathrm{DIM}_{i}+\mathrm{DIM}_{j}\right)$.

In the second case, DIM for the parameter " $x_{i}$ " is the fraction of the total change of the system unavailability, that is, due to the changes of parameters, taken one at a time. DIM for the component " $i$ " can be estimated as the sum of DIM for the parameters that specify its unavailability model. For instance, by assuming the exponential distribution for the failure and repair times $U\left(\ldots, U_{i}\left(\lambda_{i}, \mu_{i}\right), \ldots\right)$, DIM for the component " $i$ " turns out to be the following:

$$
\begin{aligned}
\operatorname{DIM}_{i} & =\operatorname{DIM}_{\lambda_{i}}+\operatorname{DIM}_{\mu_{i}}, \quad \text { where } \\
\operatorname{DIM}_{\lambda_{i}} & =\frac{\left(\partial U / \partial \lambda_{i}\right) \cdot d \lambda_{i}}{\sum_{j=1}^{n}\left(\left(\partial U / \partial \lambda_{j}\right) \cdot d \lambda_{j}+\left(\partial U / \partial \mu_{j}\right) \cdot d \mu_{j}\right)} \\
\operatorname{DIM}_{\mu_{i}} & =\frac{\left(\partial U / \partial \mu_{i}\right) \cdot d \mu_{i}}{\sum_{j=1}^{n}\left(\left(\partial U / \partial \lambda_{j}\right) \cdot d \lambda_{j}+\left(\partial U / \partial \mu_{j}\right) \cdot d \mu_{j}\right)} .
\end{aligned}
$$

The estimation of DIM for the component " $i$ " through (6) allows to consider separately the importance of the failure and repair processes but requires the computation of more and more complex partial derivatives of the system unavailability, with respect to each parameter, for each component.

Generally, the measures estimated through (5) and (6) assume different values because of different assumptions (change of components unavailability and change of parameters, resp.). Looking at the unavailability of differential $d U_{i}=$ $\left(\partial U_{i} / \partial \lambda_{i}\right) d \lambda_{i}+\left(\partial U_{i} / \partial \mu_{i}\right) d \mu_{i}$, the measures coincide only if the parameters changes $(d \lambda$ and $d \mu)$ provide the same components unavailability changes $\left(d U_{i}=d U_{j}\right.$, for all $\left.i, j\right)$ or percentage changes $\left(d U_{i} / U_{i}=d U_{j} / U_{j}\right.$, for all $\left.i, j\right)$.
It must be remarked that the opportunity to consider different changes of input variables (e.g., through the computation of DIM for Markov models by the Perturbation Analysis [11]) is very interesting in the estimation of high order measures but it is not relevant for the first order DIM which deals with "small" changes of variables.

\section{Techniques and Models}

3.1. DIM for Repairable Components. We refer to a system made by binary repairable components, and we assume the exponential distribution for their failure and repair times. Components are "as good as new" after corrective maintenance. Input variables are independent (i.e., there is not any relationship among the components unavailability $U_{i} /$ parameters $\lambda_{i}, \mu_{i}$ ).

Because of the homogeneity of measure units of parameters, both hypotheses, uniform changes and uniform percentage changes of parameters, could be used [8]. Looking at the unavailability of differential $d U_{i}=\left(\partial U_{i} / \partial \lambda_{i}\right) \cdot d \lambda_{i}+\left(\partial U_{i} / \partial \mu_{i i}\right)$. $d \mu_{i}, d U_{i} \neq 0$ if and only if $\left|d \mu_{i} / \mu_{i}\right| \neq\left|d \lambda_{i} / \lambda_{i}\right|$. Therefore, DIM can be estimated under the uniform changes of parameters $\left(d \lambda_{i}=d \mu_{i}=d \lambda_{j}=d \mu_{j}\right.$, for all $\left.i, j\right)$ only.

By (6), it is possible to separate the dependence of the system unavailability on components unavailability from the dependence of each component unavailability on parameters that specify its failure and repair probability distributions. The first-order partial derivatives of the component unavailability in (1) have been analytically estimated with respect to each parameter $(\lambda, \mu)$ and introduced in (6).

DIM for parameters $\lambda_{i}$ and $\mu_{i}$ turns out to be the following:

$$
\begin{aligned}
\operatorname{DIM}_{\lambda_{i}} & =\frac{\left(\partial U / \partial U_{i}\right) \cdot\left(\partial U_{i} / \partial \lambda_{i}\right)}{\sum_{j=1}^{n}\left(\partial U / \partial U_{j}\right) \cdot\left(\partial U_{j} / \partial \lambda_{j}+\partial U_{j} / \partial \mu_{j}\right)} \\
& =\frac{B_{i} \cdot \Delta_{\lambda_{i}}}{\sum_{j=1}^{n} B_{j} \cdot \Delta_{j}}, \quad \text { where } \Delta_{\lambda_{i}}=\frac{\mu_{i}}{\left(\mu_{i}+\lambda_{i}\right)^{2}}, \\
\operatorname{DIM}_{\mu_{i}} & =\frac{\left(\partial U / \partial U_{i}\right) \cdot\left(\partial U / \partial \mu_{i}\right)}{\sum_{j=1}^{n}\left(\partial U / \partial U_{j}\right) \cdot\left(\partial U_{j} / \partial \lambda_{j}+\partial U_{j} / \partial \mu_{j}\right)} \\
& =\frac{B_{i} \cdot \Delta_{\mu_{i}}}{\sum_{j=1}^{n} B_{j} \cdot \Delta_{j}}, \quad \text { where } \Delta_{\mu_{i}}=-\frac{\lambda_{i}}{\left(\mu_{i}+\lambda_{i}\right)^{2}} .
\end{aligned}
$$

DIM for the component " $i$ " is related to the contemporary changes of all its parameters. Because of DIM additivity property, it turns out to be the following:

$$
\begin{aligned}
& \operatorname{DIM}_{i}=\frac{B_{i} \cdot \Delta_{i}}{\sum_{j=1}^{n} B_{j} \cdot \Delta_{j}} \text {, where } \\
& \Delta_{j}=\Delta_{\lambda_{j}}+\Delta_{\mu_{j}}=\frac{\mu_{j}-\lambda_{j}}{\left(\mu_{j}+\lambda_{j}\right)^{2}} .
\end{aligned}
$$

DIM for components and parameters can be estimated through (7) and (8), on the basis of the Birnbaum measure 
for components, which only depends on the system function and can be estimated through (4).

This approach aims to consider separately the nonlinearity of the relationship between the unavailability of the system and its components, neglected in the first-order approximation of the $U\left(\ldots, U_{i}, \ldots\right)$, and the nonlinearity between the component unavailability and its parameters, neglected in the evaluation of $\Delta_{i}=\Delta_{\lambda_{i}}+\Delta_{\mu_{i}}$.

The comparison between (5) and (8) clarifies the different values of the measure, due to the changes of the components unavailability and of their parameters.

3.2. DIM for Components Subjected to Aging Phenomena. Aging phenomena are introduced into the unavailability models of repairable components by assuming the exponential-linear or the Weibull distribution for their failure times. In the first case, we consider two different assumptions for the maintenance period. The first "basic" assumption is taken from paper [8]; the second "alternative" assumption is proposed in order to acknowledge that preventive maintenance is effective only with reference to aging phenomena. In all cases (exponential-linear distribution with basic and alternative assumptions and Weibull distribution), we provide analytical equations that allow the estimation of the first-order Differential Importance Measures for components and parameters, on the basis of the Birnbaum measure for components, which only depends on the system function and can be estimated through (4).

3.2.1. Exponential-Linear Distribution. Under the same general assumptions, aging phenomena are introduced into the model by assuming the exponential-linear distribution for the components failure times.

A "Basic" assumption is made for the Maintenance period: according to [8], the Maintenance Period is specified for each component as a fraction of its "effective Mean Time To Failure", which is the inverse of its effective failure rate. By (2), we have the following results:

$$
\begin{aligned}
\tau_{i}= & \frac{1}{\alpha} \cdot \frac{1}{\lambda_{i}^{*}}=\frac{1}{\alpha} \cdot \frac{1}{\left(\lambda_{0 i}+(1 / 2) a_{i} \cdot \tau_{i}\right)} \longrightarrow \tau_{i}=\frac{\lambda_{0 i}}{a_{i}} \\
& \cdot\left(\sqrt{1+\frac{1}{\alpha \cdot\left(\lambda_{0 i}^{2} /\left(2 \cdot a_{i}\right)\right)}}-1\right) .
\end{aligned}
$$

Equation (9) constrains the maintenance period $\tau_{i}$ to the remaining parameters influencing the component failure probability $\left(\lambda_{0 i}, a_{i}\right)$.

According to [8], we assume that $\alpha$ is a known (fixed) parameter, and we are interested in the importance ranking of the remaining ones $\left(\lambda_{0 i}, a_{i}\right.$, and $\left.\mu_{i}\right)$. We assume $\alpha>1$ in compliance with the hypothesis that the component failure rate increases only slightly during the maintenance period.

In order to estimate the Differential Importance Measure for parameters and components, (9) has been introduced into (2) and then into (1); the first-order partial derivatives of the component unavailability have been analytically estimated with respect to each parameter. DIM is evaluated under the hypothesis of uniform percentage changes of parameters $\left(d \lambda_{0 i} / \lambda_{0 i}=d a_{i} / a_{i}=d \mu_{i} / \mu_{i}=d \lambda_{0 j} / \lambda_{0 j}=d a_{j} / a_{j}=d \mu_{j} / \mu_{j}\right.$, for all $i, \mathrm{j})$.

DIM for the component " $i$ " is $\operatorname{DIM}_{i}=\left(B_{i} \cdot \Delta_{i}^{I}\right) /\left(\sum_{j=1}^{n} B_{j}\right.$. $\left.\Delta_{j}^{I}\right)$, where $\tau(\lambda, a, \alpha)$ is specified by (9) and

$$
\begin{aligned}
\Delta_{j}^{I} & =\frac{\partial U_{j}}{\partial \lambda_{0 j}} \cdot \lambda_{0 j}+\frac{\partial U_{j}}{\partial a_{j}} \cdot a_{j}+\frac{\partial U_{j}}{\partial \mu_{j}} \cdot \mu_{j} \\
& =\left(-\sqrt{\frac{a_{j}^{2} \cdot \mu_{j}^{2}}{4 \cdot \alpha \cdot\left(\alpha \cdot \lambda_{0 j}^{2}+2 \cdot a_{j}\right)}}\right) \cdot\left(\lambda_{0 j}+\frac{a_{i} \cdot \tau_{i}}{2}+\mu_{j}\right)^{-2} .
\end{aligned}
$$

DIM for the component " $i$ " can be estimated as the sum of DIM for its parameters, which are computed through the following equations:

$$
\begin{aligned}
& \operatorname{DIM}_{\lambda_{0 i}}=B_{i} \cdot \lambda_{0 i} \cdot\left[\frac{\mu_{i}}{2} \cdot\left(1+\sqrt{\frac{\alpha \cdot \lambda_{0 i}^{2}}{\alpha \cdot \lambda_{0 i}^{2}+2 \cdot a_{i}}}\right)\right. \\
& \left.\cdot\left(\lambda_{0 i}+\frac{a_{i} \cdot \tau_{i}}{2}+\mu_{i}\right)^{-2}\right] \\
& {\left[\sum_{j=1}^{n} B_{j} \cdot \Delta_{j}^{I}\right]^{-1}} \\
& \operatorname{DIM}_{a_{i}}=B_{i} \cdot a_{i} \cdot\left[\frac { \mu _ { i } } { 2 } \cdot \left(\tau+\frac{\lambda_{0 i}}{a_{i}}\right.\right. \\
& \left.-\sqrt{\frac{\left(\alpha \cdot \lambda_{0 i}^{2}+a_{i}\right)^{2}}{a_{i}^{2} \cdot \alpha \cdot\left(\alpha \cdot \lambda_{0 i}^{2}+2 \cdot a_{i}\right)}}\right) \\
& \left.\cdot\left(\lambda_{0 i}+\frac{a_{i} \cdot \tau_{i}}{2}+\mu_{i}\right)^{-2}\right] \\
& \cdot\left[\sum_{j=1}^{n} B_{j} \cdot \Delta_{j}^{I}\right]^{-1} \\
& \operatorname{DIM}_{\mu_{i}}=B_{i} \cdot \mu_{i} \cdot\left[-\left(\lambda_{0 i}+\frac{a_{i} \cdot \tau_{i}}{2}\right)\right. \\
& \left.\cdot\left(\lambda_{0 i}+\frac{a_{i} \cdot \tau_{i}}{2}+\mu_{i}\right)^{-2}\right] \\
& \cdot\left[\sum_{j=1}^{n} B_{j} \cdot \Delta_{j}^{I}\right]^{-1} \cdot
\end{aligned}
$$


Then, an "alternative" assumption is made for the maintenance period. According to (9), it assumes a finite value also without aging phenomena $\left(\tau \stackrel{a \rightarrow 0}{\longrightarrow}\left(\alpha \cdot \lambda_{0}\right)^{-1}\right)$. In order to acknowledge that preventive maintenance is effective only in presence of aging phenomena, we assume that the maintenance period is a fraction of the inverse of the timedependent term of the component failure rate. From (2), we have the following results:

$$
\tau_{i}=\frac{1}{\alpha} \cdot \frac{1}{\left(\left(a_{i} \cdot \tau_{i}\right) / 2\right)} \longrightarrow \tau_{i}=\sqrt{\frac{2}{\alpha \cdot a_{i}}} .
$$

According to (15), the maintenance period does not depend on the constant term of the component failure rate and increases (indefinitely) with the decreasing of the aging phenomena $(\tau \stackrel{a \rightarrow 0}{\longrightarrow} \infty)$. As in the previous case, we assume $\alpha>1$. In order to estimate the Differential Importance Measure for parameters and components, (15) has been introduced into (2) and then into (1); the firstorder partial derivatives of the component unavailability have been analytically estimated with respect to each parameter. DIM is evaluated under the hypothesis of uniform percentage changes of parameters $\left(d \lambda_{0 i} / \lambda_{0 i}=d a_{i} / a_{i}=d \mu_{i} / \mu_{i}=\right.$ $d \alpha_{i} / \alpha_{i}=d \lambda_{0 j} / \lambda_{0 j}=d a_{j} / a_{j}=d \mu_{j} / \mu_{j}=d \alpha_{j} / \alpha_{j}$, for all $i$, $j)$.

DIM for the component " $i$ ” is $\operatorname{DIM}_{i}=\left(B_{i} \cdot \Delta_{i}^{I I}\right) /\left(\sum_{j=1}^{n} B_{j}\right.$. $\Delta_{j}^{I I}$ ) where

(i) by considering all parameters as unknown (unfixed) ones,

$$
\begin{aligned}
\Delta_{j}^{I I} & ={ }^{*} \Delta_{j}^{I I}=\frac{\partial U_{j}}{\partial \lambda_{0 j}} \cdot \lambda_{0 j}+\frac{\partial U_{j}}{\partial a_{j}} \cdot a_{j}+\frac{\partial U_{j}}{\partial \mu_{j}} \cdot \mu_{j}+\frac{\partial U_{j}}{\partial \alpha_{j}} \cdot \alpha_{j} \\
& =\left(-\sqrt{\frac{a_{j}^{2} \cdot \mu_{j}^{2}}{2 \cdot \alpha_{j} \cdot a_{j}}}\right) \cdot\left(\lambda_{0 j}+\sqrt{\frac{a_{j}}{2 \cdot \alpha_{j}}}+\mu_{j}\right)^{-2} ;
\end{aligned}
$$

(ii) by assuming that $\alpha_{i}=\alpha$, for all $i$, is a known (fixed) parameter,

$$
\begin{aligned}
\Delta_{j}^{I I} & ={ }^{* *} \Delta_{j}^{I I}=\frac{\partial U_{j}}{\partial \lambda_{0 j}} \cdot \lambda_{0 j}+\frac{\partial U_{j}}{\partial a_{j}} \cdot a_{j}+\frac{\partial U_{j}}{\partial \mu_{j}} \cdot \mu_{j} \\
& =\left(-\sqrt{\frac{a_{j}^{2} \cdot \mu_{j}^{2}}{8 \cdot \alpha \cdot a_{j}}}\right) \cdot\left(\lambda_{0 j}+\sqrt{\frac{a_{j}}{2 \cdot \alpha}}+\mu_{j}\right)^{-2} .
\end{aligned}
$$

Equivalently, DIM for components can be estimated as the sum of DIM for its (unfixed) parameters, which are computed through the following equations:

$$
\begin{aligned}
\operatorname{DIM}_{\lambda_{0 i}}= & B_{i} \cdot \lambda_{0 i} \cdot\left[\mu_{i} \cdot\left(\lambda_{0 i}+\sqrt{\frac{a_{i}}{2 \cdot \alpha_{i}}}+\mu_{i}\right)^{-2}\right] \\
& \cdot\left[\sum_{j=1}^{n} B_{j} \cdot \Delta_{j}^{I I}\right]^{-1}, \\
\operatorname{DIM}_{a_{i}=} B_{i} \cdot a_{i} \cdot\left[\frac{\mu_{i}}{2} \cdot\left(\tau_{i}-\sqrt{\frac{1}{2 \cdot \alpha_{i} \cdot a_{i}}}\right)\right. & \left.\cdot\left(\lambda_{0 i}+\sqrt{\frac{a_{i}}{2 \cdot \alpha_{i}}}+\mu_{i}\right)^{-2}\right] \\
& \left.\cdot \sum_{j=1}^{n} B_{j} \cdot \Delta_{j}^{I I}\right]^{-1}, \\
\operatorname{DIM}_{\mu_{i}}= & B_{i} \cdot \mu_{i} \cdot\left[-\left(\lambda_{0 i}^{n} B_{j} \cdot \Delta_{j}^{I I}\right]^{-1} \cdot \sqrt{\frac{a_{i}}{2 \cdot \alpha_{i}}}\right) \\
& \cdot\left[\sum_{j=1}^{n} B_{j} \cdot \Delta_{j}^{I I}\right]^{-1}, \\
\operatorname{DIM}_{\alpha_{i}}= & B_{i} \cdot \alpha_{i} \cdot\left[-\frac{a_{i} \cdot \mu_{i}}{4 \alpha_{i}} \cdot \sqrt{\frac{2}{\alpha_{i} \cdot a_{i}}}\right. \\
\left.\cdot\left(\lambda_{0 i}+\sqrt{\frac{a_{i}}{2 \cdot \alpha_{i}}}+\mu_{i}\right)^{-2}\right] & \\
& \\
& {\left.\left[\lambda_{0 i}+\sqrt{\frac{a_{i}}{2 \cdot \alpha_{i}}}+\mu_{i}\right)^{-2}\right] }
\end{aligned}
$$

DIM for parameters provides information about the Importance of random failure $\left(\mathrm{DIM}_{\lambda_{0}}\right)$, aging phenomena $\left(\operatorname{DIM}_{a}\right)$, and corrective $\left(\operatorname{DIM}_{\mu}\right)$ and preventive $\left(\operatorname{DIM}_{\alpha}\right)$ maintenance. DIM for parameters can be added over the components in order to estimate the importance of each kind of parameter $\left(\operatorname{DIM}_{\lambda_{0}, a, \mu, \alpha}=\sum_{i=1}^{n} \operatorname{DIM}_{\lambda_{0 i}, a_{i}, \mu_{i}, \alpha_{i}}\right)$. 
3.2.2. Weibull Distribution. By (3), assuming that the maintenance period is a fraction of the inverse of the timedependent term of the effective failure rate, we have the following results:

$$
\tau_{i}=\frac{1}{\alpha} \frac{1}{\gamma_{i}^{\beta_{i}} \tau_{i}^{\beta_{i}-1}} \longrightarrow \tau_{i}=\frac{1}{\gamma_{i} \cdot \alpha^{1 / \beta_{i}}} .
$$

Equation (19) constrains the maintenance period $\tau$ to the remaining parameters influencing the component failure probability $\left(\gamma_{i}, \beta_{i}\right)$. For $\beta=2$ and $\gamma=\sqrt{a / 2}$, with $\alpha>1$, (15) and (19) coincide.

In order to estimate the Differential Importance Measure for parameters and components, (19) has been introduced into (3) and then into (1); the first-order partial derivatives of the component unavailability have been analytically estimated with respect to each parameter.

DIM is evaluated under the hypothesis of uniform percentage changes of parameters $\left(d \lambda_{0 i} / \lambda_{0 i}=d \gamma_{i} / \gamma_{i}=d \beta_{i} / \beta_{i}=\right.$ $d \mu_{i} / \mu_{i}=d \alpha_{i} / \alpha_{i}=d \lambda_{0 j} / \lambda_{0 j}=d \gamma_{j} / \gamma_{j}=d \beta_{j} / \beta_{j}=d \mu_{j} / \mu_{j}=$ $d \alpha_{j} / \alpha_{j}$, for all $\left.i, j\right)$.

DIM for the component " $i$ " is $\operatorname{DIM}_{i}=\left(B_{i} \cdot \Delta_{i}^{I I I}\right) /\left(\sum_{j=1}^{n} B_{j}\right.$. $\left.\Delta_{j}^{I I I}\right)$, where

(i) by considering all parameters as unknown (unfixed) ones,

$$
\begin{aligned}
\Delta_{j}^{I I I}= & { }^{*} \Delta_{j}^{I I I} \\
= & \frac{\partial U_{j}}{\partial \lambda_{0 j}} \cdot \lambda_{0 j}+\frac{\partial U_{j}}{\partial \gamma_{j}} \cdot \gamma_{j}+\frac{\partial U_{j}}{\partial \beta_{j}} \cdot \beta_{j}+\frac{\partial U_{j}}{\partial \mu_{j}} \cdot \mu_{j}+\frac{\partial U_{j}}{\partial \alpha_{j}} \cdot \alpha_{j} \\
= & \left(-\frac{\gamma_{j} \cdot \mu_{j}}{\beta_{j}} \cdot \alpha_{j}^{\left(1 / \beta_{j}\right)-1} \cdot \ln \alpha_{j}+\gamma_{j} \cdot \mu_{j}\right. \\
& \left.\cdot\left(\frac{1}{\beta_{j}}-1\right) \cdot \alpha_{j}^{\left(1 / \beta_{j}\right)-1}\right) \\
& \cdot\left(\lambda_{0 j}+\gamma_{j} \cdot \alpha_{j}^{\left(1 / \beta_{j}\right)-1}+\mu_{j}\right)^{-2}
\end{aligned}
$$

(ii) by assuming that $\alpha_{i}=\alpha$, for all $i$, is a known (fixed) parameter,

$$
\begin{aligned}
\Delta_{j}^{I I I}= & { }^{* *} \Delta_{j}^{I I I} \\
= & \frac{\partial U_{j}}{\partial \lambda_{0 j}} \cdot \lambda_{0 j}+\frac{\partial U_{j}}{\partial \gamma_{j}} \cdot \gamma_{j}+\frac{\partial U_{j}}{\partial \beta_{j}} \cdot \beta_{j}+\frac{\partial U_{j}}{\partial \mu_{j}} \cdot \mu_{j} \\
= & \left(-\frac{\gamma_{j} \cdot \mu_{j}}{\beta_{j}} \cdot \alpha^{\left(1 / \beta_{j}\right)-1} \cdot \ln \alpha\right) \\
& \cdot\left(\lambda_{0 i}+\gamma_{j} \cdot \alpha^{\left(1 / \beta_{j}\right)-1}+\mu_{j}\right)^{-2} ;
\end{aligned}
$$

(iii) by assuming that $\beta_{i}=\beta$, for all $i$, is a known (fixed) parameter,

$$
\begin{aligned}
\Delta_{j}^{I I I}= & { }^{* * *} \Delta_{j}^{I I I} \\
= & \frac{\partial U_{j}}{\partial \lambda_{0 j}} \cdot \lambda_{0 j}+\frac{\partial U_{j}}{\partial \gamma_{j}} \cdot \gamma_{j}+\frac{\partial U_{j}}{\partial \mu_{j}} \cdot \mu_{j}+\frac{\partial U_{j}}{\partial \alpha_{j}} \cdot \alpha_{j} \\
= & \left(\gamma_{j} \cdot \mu_{j} \cdot\left(\frac{1}{\beta}-1\right) \cdot \alpha_{j}^{(1 / \beta)-1}\right) \\
& \cdot\left(\lambda_{0 j}+\gamma_{i} \cdot \alpha_{j}^{(1 / \beta)-1}+\mu_{j}\right)^{-2} .
\end{aligned}
$$

Equivalently, DIM for components can be estimated as the sum of DIM for its (unfixed) parameters, which are computed through the following equations:

$$
\begin{aligned}
& \operatorname{DIM}_{\lambda_{0 i}}= B_{i} \cdot \lambda_{0 i} \cdot\left[\mu_{i} \cdot\left(\lambda_{0 i}+\gamma_{i} \cdot \alpha_{i}^{\left(1 / \beta_{i}\right)-1}+\mu_{i}\right)^{-2}\right] \\
& \cdot\left[\sum_{j=1}^{n} B_{j} \cdot \Delta_{j}^{I I I}\right]^{-1}, \\
& \operatorname{DIM}_{\gamma_{i}}= B_{i} \cdot \gamma_{i} \cdot\left[\left(\mu_{i} \cdot \alpha_{i}^{\left(1 / \beta_{i}\right)-1}\right)\right. \\
& \cdot\left[\sum_{j=1}^{n} B_{j} \cdot \Delta_{j}^{I I I}\right]^{-1}, \\
&\left.\left.\cdot\left[\sum_{j=1}^{n} B_{j} \cdot \Delta_{j}^{I I I}\right]^{-1}, \alpha_{i}^{\left(1 / \beta_{i}\right)-1}+\mu_{i}\right)^{-2}\right] \\
& \operatorname{DIM}_{\beta_{i}=} B_{i} \cdot \beta_{i} \cdot\left[-\left(\frac{\gamma_{i} \cdot \mu_{i}}{\beta_{i}^{2}} \cdot \alpha_{i}^{\left(1 / \beta_{i}\right)-1} \cdot \ln \alpha_{i}\right)\right. \\
&\left.\sum_{j=1}^{n} B_{j} \cdot \Delta_{j}^{I I I}\right]^{-1}, \\
&\left.\cdot\left(\lambda_{0 i}+\gamma_{i} \cdot \alpha_{i}^{\left(1 / \beta_{i}\right)-1}+\mu_{i}\right)^{-2}\right]
\end{aligned}
$$




$$
\begin{aligned}
\operatorname{DIM}_{\alpha_{i}}= & B_{i} \cdot \alpha_{i} \cdot\left[\left(\mu_{i} \cdot \gamma_{i} \cdot\left(\frac{1}{\beta_{i}}-1\right) \cdot \alpha_{i}^{\left(1 / \beta_{i}\right)-2}\right)\right. \\
& \left.\cdot\left(\lambda_{0 i}+\gamma_{i} \cdot \alpha_{i}^{\left(1 / \beta_{i}\right)-1}+\mu_{i}\right)^{-2}\right] \\
& \cdot\left[\sum_{j=1}^{n} B_{j} \cdot \Delta_{j}^{I I I}\right]^{-1} \cdot
\end{aligned}
$$

DIM for parameters provides information about importance of random failure $\left(\mathrm{DIM}_{\lambda_{0}}\right)$, aging $\left(\mathrm{DIM}_{\gamma}\right.$ and $\left.\mathrm{DIM}_{\beta}\right)$, and corrective $\left(\mathrm{DIM}_{\mu}\right)$ and preventive $\left(\mathrm{DIM}_{\alpha}\right)$ maintenance.

Having $\left(\partial U_{j} / \partial \lambda_{0 j}\right) \cdot \lambda_{0 j}+\left(\partial U_{j} / \partial \gamma_{j}\right) \cdot \gamma_{j}+\left(\partial U_{j} / \partial \mu_{j}\right) \cdot$ $\mu_{j}=0$, it results that ${ }^{*} \Delta_{j}^{I I I}={ }^{* *} \Delta_{j}^{I I I}+{ }^{* * *} \Delta_{j}^{I I I}$; it follows that $\mathrm{DIM}_{\lambda_{0 i}}+\mathrm{DIM}_{\gamma_{i}}+\mathrm{DIM}_{\mu_{i}}=0$ and $\mathrm{DIM}_{i}=\mathrm{DIM}_{\beta_{i}}$, if $\alpha_{i}=\alpha_{j}=\alpha$ for all $i, j$ or $\operatorname{DIM}_{i}=\operatorname{DIM}_{\alpha_{i}}$ if $\beta_{i}=\beta_{j}=\beta$ for all $i, j$.

DIM for parameters can be added over components in order to estimate the importance of each kind of parameter $\left(\right.$ DIM $\left._{\lambda_{0}, \gamma, \beta, \mu, \alpha}=\sum_{i=1}^{n} \operatorname{DIM}_{\lambda_{0 i}, \gamma_{i}, \beta_{i}, \mu_{i}, \alpha_{i}}\right)$.

The above equations are consistent with the ones previously provided for the exponential-linear distribution by assuming that $\beta$ is a known (fixed) parameter and by referring to the change of the parameter $2 \cdot \gamma^{2}$. By introducing $\beta=2$ and $\gamma=\sqrt{a / 2},(23),(26)$, and (27) coincide with (15), (17), and (18). Having $d \gamma=(8 a)^{-1 / 2} d a$, DIM for the parameter $2 \cdot \gamma^{2}$ turns out to be the following:

$$
\begin{aligned}
\operatorname{DIM}_{a_{i}=2 \gamma_{i}^{2}=} B_{i} \cdot a_{i} \cdot[ & \left(\mu_{i} \cdot \alpha_{i}^{\left(1 / \beta_{i}\right)-1} \cdot \sqrt{\frac{1}{8 \cdot a_{i}}}\right) \\
& \left.\cdot\left(\lambda_{0 i}+\alpha_{i}^{\left(1 / \beta_{i}\right)-1} \cdot \sqrt{\frac{a_{i}}{2}}+\mu_{i}\right)^{-2}\right] \\
& \cdot\left[\sum_{j=1}^{n} B_{j} \cdot \Delta_{j}^{I I I}\right]^{-1} \cdot
\end{aligned}
$$
(27).

By introducing $\beta=2$ and $\gamma=\sqrt{a / 2},(28)$ coincides with

3.3. DIM and Improvement of the System Performance. The first-order Differential Importance Measure allows the ranking of the input variables, according to the fraction of total change in the model output (system unavailability), that is, due to their small, one at a time changes. DIM for parameters provides information about the importance of the random failure, aging phenomena, and corrective and preventive maintenance. DIM for components provides information about the importance of the components themselves.

Information about the effect of the parameters changes is provided by the sign of the first-order partial derivatives of the system unavailability, while the sign of DIM depends on the values of the input variables. By assuming the exponential-linear distribution for the components failure times, the increase of $\lambda_{0}$ and $a$ leads to the increase of the system unavailability $\left(\partial U / \partial \lambda_{0}\right.$ and $\left.\partial U / \partial a>0\right)$, while the increase of $\mu$ and $\alpha$ leads to the decrease of the system unavailability $(\partial U / \partial \mu$ and $\partial U / \partial \alpha<0)$. By assuming the Weibull distribution for the components failure times, the increase of $\lambda_{0}$ and $\gamma$ leads to the increase of the system unavailability $\left(\partial U / \partial \lambda_{0}\right.$ and $\left.\partial U / \partial \gamma>0\right)$, while the increase of $\beta, \mu$, and $\alpha$ leads to the decrease of the system unavailability $(\partial U / \partial \beta$ (since $\alpha<1), \partial U / \partial \mu$, and $\partial U / \partial \alpha<0)$.

The absolute values of DIM define a ranking of parameters that provides information about the effectiveness of their changes in the reduction of the system unavailability.

The improvement of the system performance (i.e., reduction of the system unavailability) can be supported by the information coming from a two-step process.

The first step aims to identify the "critical" parameters that define the failure and repair probability distributions of components, by assuming the same preventive maintenance "strategy" for all components (i.e., a fixed parameter $\alpha$ ). Generally, technological constraints limit the improvement of the reliability (reduction of random failures and/or aging) and maintainability (increase of the repair rates) performance of components.

The second step aims to support the specification of "optimal" values for the Maintenance period of different components, through a heuristic approach. Specifically, we look for the values of $\alpha_{i}$ that reduce differences among Differential Importance Measures for components.

\section{Results and Discussion}

In order to verify the correctness of the analytical equations proposed in Section 3 and to show the advantages of their use, we solve four case studies, which refer to an example proposed in paper [8]. A system made by three repairable binary components is considered. Its reliability block diagram is shown in Figure 1.

The first case study refers to repairable components without aging. In the second and third case studies, the exponential-linear distribution is assumed for the components failure times. The second case study refers to the "basic" assumption for the maintenance period, while the third case study refers to the "alternative" one. In the fourth case study, the Weibull distribution is assumed for the components failure times; the "optimal" values are looked for the parameters $\alpha_{i}$ accounting for the preventive maintenance strategy.

4.1. Case Study 1. We consider a system made by repairable components without aging phenomena. Table 1 provides input data $\left(\lambda_{i}\right.$ and $\left.\mu_{i}\right)$ and the unavailability of components $U_{i}$ estimated through (1). The system unavailability $U=U_{3}+$ $U_{1} \cdot U_{2}-U_{1} \cdot U_{2} \cdot U_{3}=0.056$ comes from the system function $\phi=\phi_{3} \oplus\left(\phi_{1} \otimes \phi_{2}\right)$.

Following the approach described in Section 3, we estimate the Birnbaum measure for components through (4). Table 1 provides the results coming from $B_{1}=U_{2}-U_{2} \cdot U_{3}$, $B_{2}=U_{1}-U_{1} \cdot U_{3}$ and $B_{3}=1-U_{1} \cdot U_{2}$. DIM is estimated through (7) and (8) under the hypothesis of uniform changes 
TABLE 1: Case study 1, data and Differential Importance Measure.

\begin{tabular}{lcccccrr}
\hline Component & $\lambda_{i}\left[\mathrm{~h}^{-1}\right]$ & $\mu_{i}\left[\mathrm{~h}^{-1}\right]$ & $U_{i}$ & $B_{i}$ & $\mathrm{DIM}_{\lambda_{i}}$ & $\mathrm{DIM}_{\mu_{i}}$ & $\mathrm{DIM}_{i}$ \\
\hline 1 & 0.0050 & 0.0200 & 0.2000 & 0.197 & 0.158 & -0.039 & 0.118 \\
2 & 0.0050 & 0.0200 & 0.2000 & 0.197 & 0.158 & -0.039 & 0.118 \\
3 & 0.0005 & 0.0300 & 0.0164 & 0.960 & 0.776 & -0.013 & 0.763 \\
\hline
\end{tabular}

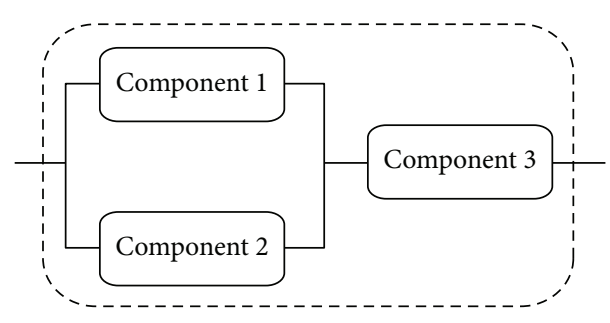

FIGURE 1: System reliability block diagram.

of parameters. Table 1 provides the obtained results. They correspond to the values estimated in paper [8].

4.2. Case Study 2. Aging phenomena are introduced into the model through the exponential-linear distribution for the components failure times. The effective value of the failure rate $\lambda^{*}$ is defined by (2). According to paper [8], the maintenance period is defined by (9).

Table $1\left(\lambda_{i}\right.$ and $\left.\mu_{i}\right)$ and Table $2\left(a_{i}\right.$ and $\left.\alpha_{i}\right)$ provide input data [8]. Table 2 also provides the "basic characteristics" of components (maintenance period, effective value of the failure rate, and unavailability) and their Birnbaum measure. By assuming the values in Table 2, the system unavailability is $U=0.205$.

DIM is estimated by assuming that $\alpha_{i}=\alpha$, for all $i$, is a known (fixed) parameter, under the hypothesis of uniform percentage changes of the remaining ones $\lambda_{0 i}, a_{i}$, and $\mu_{i}$, through (11), starting from values in Tables 1 and 2. DIM for components is the sum of DIM for parameters. Table 3 provides the obtained results.

The ranking of components and parameters defined by DIM estimated through the equations in Section 3 coincides with the ranking provided in paper [8]. Their numerical values differ, but this comparison is not significant because of different assumptions. Indeed, we explicitly consider the growth parameter $a$ and its relationships with the remaining ones, in order to have information about the importance of the random failures and aging phenomena separately; this requires the hypothesis of uniform percentage changes.

In paper [8], the growth parameter $a$ is not explicitly introduced into the model; the measure is estimated starting from the partial derivatives of the system unavailability with respect to the effective value of the components failure rates, under the hypothesis of their uniform changes. Moreover, the comparison between the ranking of components for the case studies 1 and 2 is not significant because the system unavailability differs significantly (even if some data are the same) and more generally, because of different hypotheses about (uniform/uniform percentage) changes.
4.3. Case Study 3. We assume the exponential-linear distribution for the components failure times, and we recognize that preventive maintenance is effective only in presence of aging phenomena; the maintenance period is provided by (12).

In order to evaluate the effects of the two different assumptions for the maintenance period, we consider the same data of the case study 2. The different values of the parameter $\alpha$ lead to about the system unavailability in the two Case studies.

The only difference regarding the parameter $\alpha$, is shown in Table 4; the value 1.75 is assumed; the system unavailability is about the same as that in the case study 2. Table 4 also provides the "basic characteristics" of components (maintenance period, effective value of the failure rate, and unavailability) and their Birnbaum measures.

The comparison between Table 2 (case study 2) and Table 4 (case study 3) clarifies the effects of the different assumptions for the maintenance period. By referring the maintenance period to the time-dependent term of the failure rate, it increases for the components 1 and 2, producing the increase of their effective failure rates and unavailability; it decreases for the component 3 (heavier aging phenomena), producing the decrease of its effective failure rate and unavailability.

DIM is estimated by assuming that $\alpha_{i}=\alpha$, for all $i$, is a known (fixed) parameter, under the hypothesis of uniform percentage changes of the remaining ones. DIM for parameters $\lambda_{0 i}, a_{i}$, and $\mu_{i}$ is estimated through (15), (16), and (17), respectively, on the basis of values provided in Tables 1 , 2 , and 4. DIM for component is the sum of DIM for related parameters. Table 5 provides the obtained results.

By comparing the values provided in Table 3 (case study 2) and Table 5 (case study 3), DIM estimated under the two different assumptions for the maintenance period defines the same ranking of parameters and components. The difference between the importance of the component 3 and the components 1 and 2 decreases; in that sense, the "alternative" assumption provides an optimization in the specification of the maintenance period. For example, during a life time equal to 10 years, by assuming the values specified in Tables 2 and 4 for the maintenance periods, it is necessary to realize that approximately 2,000 operations are performed (about 666 for each component) for the "basic" assumption (case study 2); approximately 1,320 operations are performed (about 295 for components 1 and 2 and 730 for component 3) for the "alternative" assumption (case study 3 ).

The absolute values of DIM produce the following ranking of parameters: $\mu_{3}, a_{3}, \mu_{1}-\mu_{2}, \lambda_{1}-\lambda_{2}, \lambda_{3}$, and $a_{1}-a_{2}$. The more effective improvement is the increase of the repair rate of the component 3. For example, by assuming $\mu_{3}=0.095$ 
TABle 2: Case study 2, data and basic characteristics of components.

\begin{tabular}{lccccccc}
\hline Component & $\lambda_{0 i}\left[\mathrm{~h}^{-1}\right]$ & $a_{i}\left[\mathrm{~h}^{-1}\right]$ & $\alpha\left[\mathrm{h}^{-1}\right]$ & $\tau_{i}\left[\mathrm{~h}^{-1}\right]$ & $\lambda_{i}^{*}\left[\mathrm{~h}^{-1}\right]$ & $U_{i}$ & $B_{i}$ \\
\hline 1 & 0.0050 & $1.30 E-05$ & & 131.400 & $5.854 E-03$ & 0.226 \\
2 & 0.0050 & $1.30 E-05$ & 1.30 & 131.400 & $5.854 E-03$ & 0.226 \\
3 & 0.0005 & $8.00 E-05$ & & 132.566 & $5.803 E-03$ & 0.190 \\
\hline
\end{tabular}

TABLe 3: Case study 2, Differential Importance Measure.

\begin{tabular}{lcccc}
\hline Component & DIM $_{a_{i}}$ & DIM $_{\lambda_{i}}$ & DIM $_{\mu_{i}}$ & DIM $_{i}$ \\
\hline 1 & -0.060 & -0.354 & 0.475 & 0.060 \\
2 & -0.060 & -0.354 & 0.475 & 0.060 \\
3 & -0.879 & -0.083 & 1.841 & 0.879 \\
\hline Total & -1.000 & -0.791 & 2.791 & 1.000 \\
\hline
\end{tabular}

TABLE 4: Case study 3, data and basic characteristic of the components.

\begin{tabular}{lccccc}
\hline Component & $\alpha_{i}\left[\mathrm{~h}^{-1}\right]$ & $\tau_{i}^{*}\left[\mathrm{~h}^{-1}\right]$ & $\lambda_{i}^{*}\left[\mathrm{~h}^{-1}\right]$ & $U_{i}$ & $B_{i}$ \\
\hline 1 & & 296.50 & $6.93 E-03$ & 0.257 & 0.2188 \\
2 & 1.75 & 296.50 & $6.93 E-03$ & 0.257 & 0.2188 \\
3 & & 119.52 & $5.28 E-03$ & 0.150 & 0.9338 \\
\hline
\end{tabular}

TABle 5: Case study 3, Differential Importance Measure.

\begin{tabular}{lcccc}
\hline Component & $\mathrm{DIM}_{\lambda_{0 i}}$ & $\mathrm{DIM}_{a_{i}}$ & $\mathrm{DIM}_{\mu_{i}}$ & $\mathrm{DIM}_{i}$ \\
\hline 1 & -0.461 & -0.089 & 0.639 & 0.089 \\
2 & -0.461 & -0.089 & 0.639 & 0.089 \\
3 & -0.172 & -0.822 & 1.817 & 0.822 \\
\hline Total & -1.094 & -1.000 & 3.094 & 1.000 \\
\hline
\end{tabular}

TABLE 6: Case study 4, data and basic characteristics of components.

\begin{tabular}{lccccc}
\hline Component & $\gamma\left[\mathrm{h}^{-1}\right]$ & $\beta$ & $\lambda_{i}^{*}\left[\mathrm{~h}^{-1}\right]$ & $U_{i}$ & $B_{i}$ \\
\hline 1 & 0.00255 & 2.00 & $6.92 E-03$ & 0.257 & 0.2188 \\
2 & 0.00255 & 2.00 & $6.92 E-03$ & 0.257 & 0.2188 \\
3 & 0.00635 & 2.00 & $5.29 E-03$ & 0.150 & 0.9338 \\
\hline
\end{tabular}

TABLE 7: Case study 4, Differential Importance Measures.

\begin{tabular}{lccccc}
\hline Component & DIM $_{\lambda_{0 i}}$ & DIM $_{\gamma_{i}}$ & DIM $_{\beta_{i}}$ & DIM $_{\mu_{i}}$ & DIM $_{i}$ \\
\hline 1 & -0.822 & -0.317 & 0.089 & 1.138 & 0.089 \\
2 & -0.822 & -0.317 & 0.089 & 1.138 & 0.089 \\
3 & -0.306 & -2.941 & 0.823 & 3.247 & 0.823 \\
\hline Total & -1.950 & -3.574 & 1.000 & 5.523 & 1.000 \\
\hline
\end{tabular}

(from $\mu_{3}=0.03$ ), the system unavailability is equal to $U=$ 0.115 (reduced from $U=0.206$ ). It results that $\mathrm{DIM}_{1}=$ $\mathrm{DIM}_{2}=0.190$ and $\mathrm{DIM}_{3}=0.619$; a further improvement should be performed if it is possible. By assuming $\mu_{3}=0.32$, the system unavailability is further reduced $(U=0.081)$ and DIM for components is about the same $\left(\mathrm{DIM}_{1}=\mathrm{DIM}_{2}=\right.$ $\left.\mathrm{DIM}_{3}=0.333\right)$.

DIM for parameters can be added over components, in order to estimate the "overall" importance of random failure, aging phenomena, and corrective and preventive maintenance. With reference to Table 5, the most important parameter is the repair rate $\mu$; the growth parameter $a$ and the constant term $\lambda_{0}$ have about the same importance.

In order to verify the correctness of the equations proposed in Section 3, the case study 3 was solved numerically. A fictitious uncertainty is assigned to parameters by a normal distribution with a "small enough" standard deviation. 1,000 trials were performed through the Latin Hypercube sampling technique. The simulation gives pairs of values for the uncertain parameter (independent variable) and for the component unavailability (dependent variable). The partial derivatives of the component unavailability with respect to each parameter have been estimated through a linear regression of data. Values obtained for the partial derivatives and then for the Differential Importance Measure coincide with the values obtained from the analytical equations proposed in Section 3.

4.4. Case Study 4. We introduce aging phenomena through the Weibull distribution for the components failure times. The maintenance period is provided by (19). Table 6 provides the input data $\left(\gamma_{i}, \beta_{i}\right)$, the values estimated for the "basic characteristics" of components (maintenance period, effective value of the failure rate, and unavailability), and their Birnbaum measure. $\lambda_{0 i}, \mu, \alpha_{i}$, and $\tau_{i}^{*}$ assume the same values introduced for the case study 3. Having $\beta=2$ and $\gamma=\sqrt{a / 2}$, components and system unavailability for the case study 3 (exponential-linear distribution) and for the case study 4 (Weibull distribution) coincide.

DIM is estimated by assuming that $\alpha_{i}=\alpha$, for all $i$, is a known (fixed) parameter, under the hypothesis of uniform percentage changes of the remaining ones. DIM for parameters $\lambda_{0 i}, \gamma_{i}, \beta_{i}$, and $\mu_{i}$ is estimated through (23), (24), (25), and (26), on the basis of values provided in Table 6. DIM for component is the sum of DIM for related parameters. Table 7 provides the obtained results.

In order to verify the correctness of the equations proposed in Section 3, the case study 4 has been solved numerically; the same approach described for the previous case study has been adopted with positive results.

If technological constraints prevent the improvement of components unavailability by changing the parameters that define their failure $\left(\lambda_{0 i}, \gamma_{i}, \beta_{i}\right)$ and repair $\left(\mu_{i}\right)$ probability distributions, the only way to reduce the system unavailability concerns the optimization of the preventive maintenance. To this purpose, we consider the parameter $\alpha$ as unfixed, we refer it to each component, and we look for the values that minimize the differences among the Differential Importance measure for the parameters $\alpha_{i}$.

By assuming $\alpha_{1}=1, \alpha_{2}=1$, and $\alpha_{3}=104$, (12) provides the maintenance periods $\tau_{1} \approx 392, \tau_{2} \approx 392$, and $\tau_{3} \approx 15.5$, 
TAble 8: Case study 4, Differential Importance Measures for $\beta_{i}=\beta_{j}=\beta$ for all $i, j$.

\begin{tabular}{|c|c|c|c|c|c|}
\hline Component & $\operatorname{DIM}_{\lambda_{0 i}}$ & $\operatorname{DIM}_{a=2 \cdot \gamma_{i}^{2}}$ & $\operatorname{DIM}_{\mu_{i}}$ & $\operatorname{DIM}_{\alpha_{i}}$ & $\mathrm{DIM}_{i}$ \\
\hline 1 & -0.231 & -0.044 & 0.319 & 0.044 & 0.089 \\
\hline 2 & -0.231 & -0.044 & 0.319 & 0.044 & 0.089 \\
\hline 3 & -0.086 & -0.411 & 0.908 & 0.411 & 0.822 \\
\hline Total & -0.547 & -0.500 & 1.547 & 0.500 & 1.000 \\
\hline
\end{tabular}

and (27) provides $\operatorname{DIM}_{\alpha_{i}} \approx 0.131$, for all $i$. It results that $\mathrm{DIM}_{1}=\mathrm{DIM}_{2}=0.131$ and $\mathrm{DIM}_{3}=0.738$.

If we assume that $\beta_{i}=\beta$, for all $i$, is a known (fixed) parameter (i.e., we remove the uncertainty on the shape parameter of the Weibull distribution; for example, we assume an exponential-linear distribution for the components failure times), it results $\mathrm{DIM}_{\alpha_{i}}=\mathrm{DIM}_{i} \approx 0.333$, for all $i$ (having $\operatorname{DIM}_{\lambda_{0 i}}+\operatorname{DIM}_{\gamma_{i}}+\operatorname{DIM}_{\mu_{i}}=0$ ).

A Last Remark. The values of DIM for parameters provided in Table 5 (case study 3, exponential-linear) and in Table 7 (case study 4, Weibull distribution) differ, while DIM for components (which is the sum of DIM for parameters) coincides. Table 8 provides the DIM for parameters $\lambda_{0 i}, a_{i}=$ $2 \cdot \gamma_{i}^{2}, \mu_{i}$, and $\alpha_{i}$ as well as DIM for components by assuming that $\beta_{i}=\beta$, for all $i$, is a known (fixed) parameter. The same values of DIM for parameters come from (15), (16), (17), and (18) proposed for exponential-linear distribution and from (23), (28), (26), and (27) proposed for Weibull distribution.

\section{Conclusions}

This paper refers to systems made by repairable binary components and subjected to aging phenomena and "perfect" maintenance, by assuming the exponential, the exponentiallinear, or the Weibull distribution for their failure times. According to paper [8], "effective values" were taken for the components failure rates, by assuming that they increase only slightly during the time maintenance period. Differently, we proposed to recognize that preventive maintenance is effective only in presence of aging phenomena.

Generally, the estimation of DIM for components requires the computation of the first-order partial derivatives of the system unavailability with respect to the components unavailability (Birnbaum measure), which only depends on the system function. The estimation of DIM for parameters requires the computation of more and more complex partial derivatives of the system unavailability (for each parameter of each component).

In this paper, we proposed to consider separately the dependence of the system unavailability on the components unavailability, from the dependence of the component unavailability on the parameters that specify its failure and repair probability distributions. Under the assumptions of independent components and "perfect" maintenance, this approach leads to the analytical equations provided in Section 3. They allow the estimation of the first-order Differential Importance Measures for components and parameters, under the hypothesis of uniform percentage changes of parameters, on the basis of the Birnbaum measure for components.

Through the proposed equations, the estimation of DIM for parameters does not require further information than that used for the estimation of DIM for components and allows considering separately the importance of the random failure, aging phenomena, and preventive and corrective maintenance. This information supports the improvement of the system performance.

We solved some case studies in order to verify the correctness of the analytical equations and to show the advantages of their use.

Results coming from the analytical equations (numeric values of the measure and ranking of parameters and components) were compared with values taken from paper [8] and coming from the numerical solution of the case studies; the comparison confirmed the consistency of the proposed equations.

Results coming from the case studies (2 and 3) show that preventive maintenance can be optimized (i.e., the maintenance period can be increased) by recognizing that it is effective only in presence of aging phenomena.

Results coming from the last case study (4) show the opportunity to apply a two-step process for the improvement of the system performance. The first step regards the improvement of the reliability (reduction of random failure and/or aging phenomena) and maintainability (increase of the repair rate) performance of components, within the applicable technological limits. The second step regards the specification of the "optimal" strategy for preventive maintenance (i.e., values of the maintenance periods that reduce differences among values of DIM).

The implementation of the proposed analytical equations into a software tool will allow their application to more complex and realistic systems.

\section{Abbreviations}

a: $\quad$ Growth parameter for an exponential-linear distribution

$B_{i}$ : $\quad$ Birnbaum measure for the component " $i$ "

Cdf: Cumulative density function

$\mathrm{DIM}_{i}$ : Differential Importance Measure for the component " $i$ "

DIM $_{X_{i}}$ : Differential Importance Measure for the parameter $X$ of the component " $i$ "

$\mu: \quad$ (Constant) repair rate

$\lambda(t): \quad$ Failure rate

$\lambda^{*}: \quad$ Effective value of the failure rate 
$\lambda_{0}$ : Initial value of the failure rate

$\beta$ : Shape parameter for a Weibull distribution

$1 / \gamma: \quad$ Scale parameter for a Weibull distribution

$\phi\left(\ldots, \phi_{i}, \ldots\right)$ : System function

Pdf: $\quad$ Probability density function

PRA: $\quad$ Probabilistic risk assessment

RAMS: Reliability, availability, maintainability, and safety

$\tau: \quad$ Maintenance period

U: Unavailability.

\section{Acknowledgments}

This study has been performed within the Project Support System for the Availability Analysis of Products and Processes (SSAAPP), partially financed by the Emilia Romagna region (Italy) and developed by NIER Ingegneria with the support of the Department of Energy, Nuclear and Environmental Control Engineering (DIENCA) of the University of Bologna.

\section{References}

[1] E. Zio and L. Podofillini, "Accounting for components interactions in the differential importance measure," Reliability Engineering \& System Safety, vol. 91, no. 10-11, pp. 1163-1174, 2006.

[2] M. C. Cheock, G. W. Parry, and R. R. Sherry, "Use of importance measures in risk-informed regulatory applications," Reliability Engineering \& System Safety, vol. 60, pp. 213-226, 1998.

[3] E. Borgonovo, "The reliability importance of components and prime implicants in coherent and non-coherent systems including total-order interactions," European Journal of Operational Research, vol. 204, no. 3, pp. 485-495, 2010.

[4] L. W. Birnbaum, "On the importance of different elements in a multi-elements system," in Multivariate Analysis, Academic Press, New York, NY, USA, 1969.

[5] X. R. Cao and H. F. Chen, "Pertubation realization, potentials, and sensitivity analysis of Markov processes," IEEE Transactions on Automatic Control, vol. 42, no. 10, pp. 1382-1393, 1997.

[6] M. Marseguerra and E. Zio, "Optimizing maintenance and repair policies via a combination of genetic algorithms and Monte Carlo simulation," Reliability Engineering \& System Safety, vol. 68, no. 1, pp. 69-83, 2000.

[7] E. Borgonovo, M. Marseguerra, and E. Zio, "A Monte Carlo methodological approach to plant availability modeling with maintenance, aging and obsolescence," Reliability Engineering \& System Safety, vol. 67, no. 1, pp. 61-73, 2000.

[8] M. Marseguerra and E. Zio, "Monte Carlo estimation of the differential importance measure: application to the protection system of a nuclear reactor," Reliability Engineering \& System Safety, vol. 86, no. 1, pp. 11-24, 2004.

[9] E. Borgonovo and G. E. Apostolakis, "A new importance measure for risk-informed decision making," Reliability Engineering \& System Safety, vol. 72, no. 2, pp. 193-212, 2001.

[10] IAEA Safety Standards, Specific Safety Guide No. SSG-3, Development and Application of Level 1 Probabilistic Safety Assessment for Nuclear Power Plants, 2010.
[11] P. Do Van, A. Barros, and C. Berenguer, "Reliability importance analysis of markovian systems at steady state using perturbation analysis," Reliability Engineering \& System Safety, vol. 93, no. 11, pp. 1605-1615, 2008. 

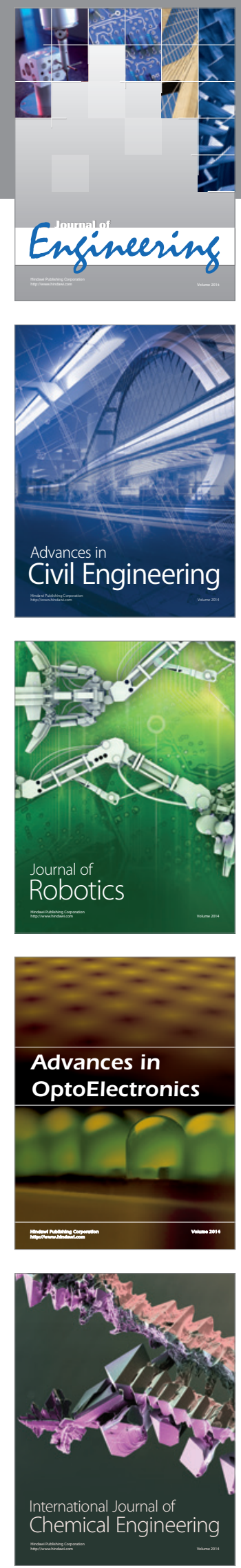

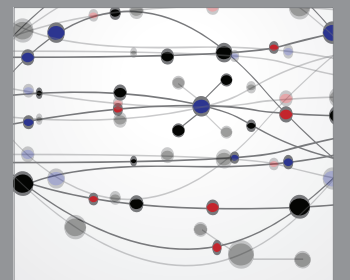

The Scientific World Journal
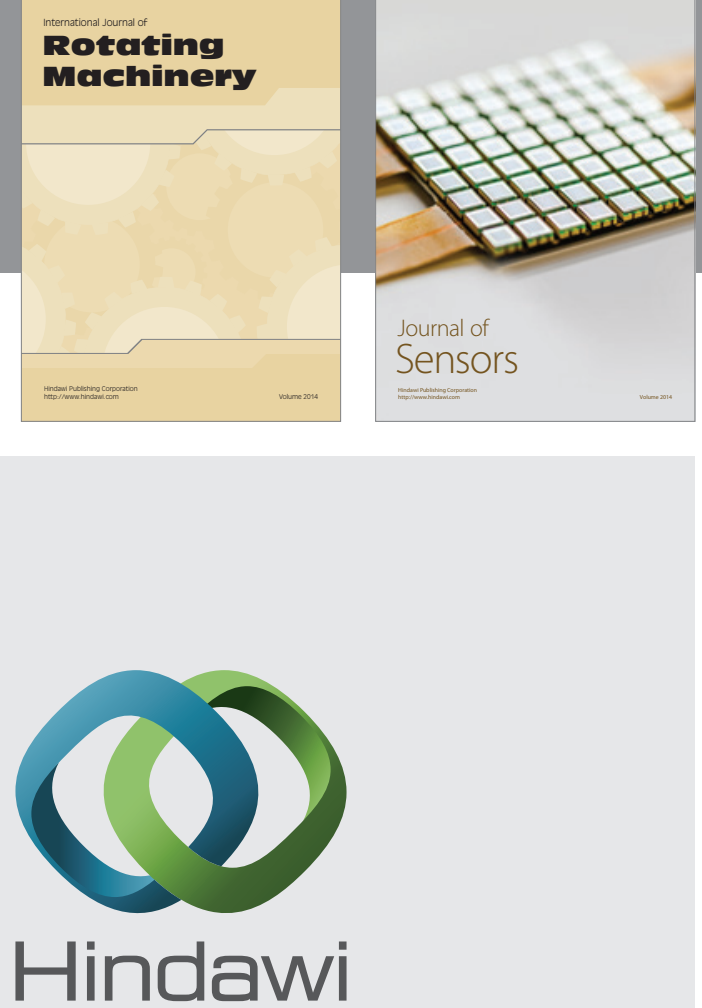

Submit your manuscripts at http://www.hindawi.com
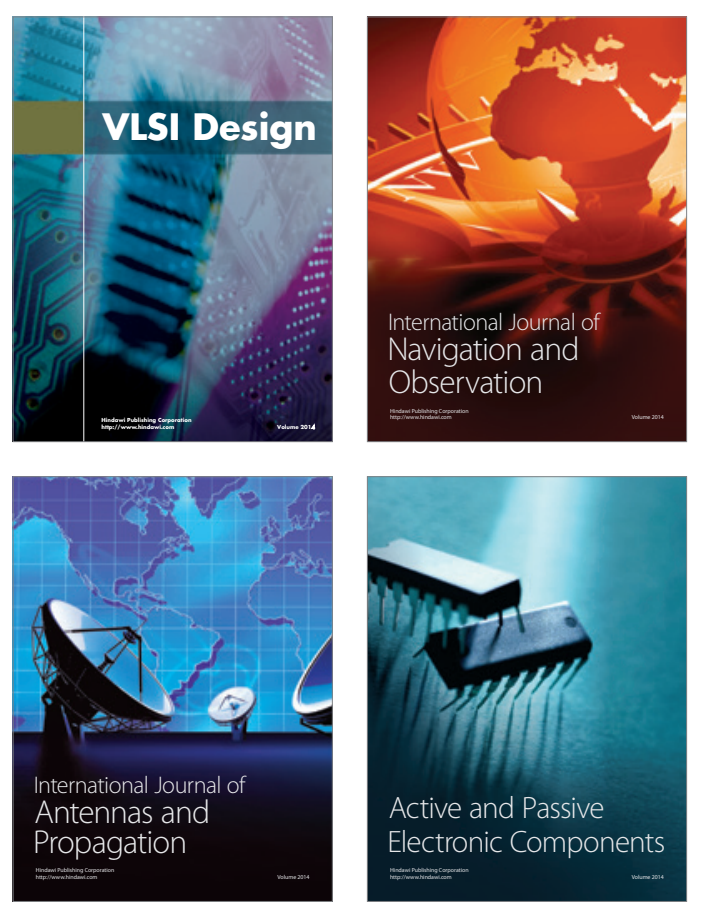
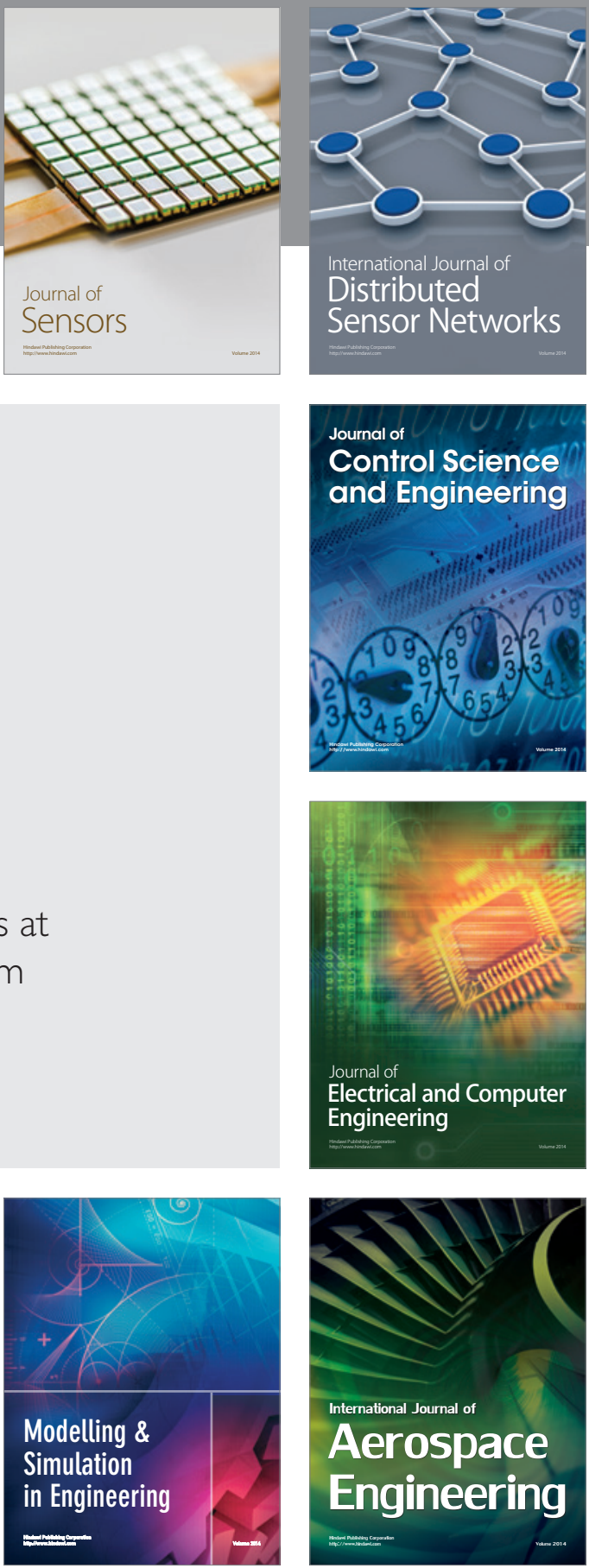

Journal of

Control Science

and Engineering
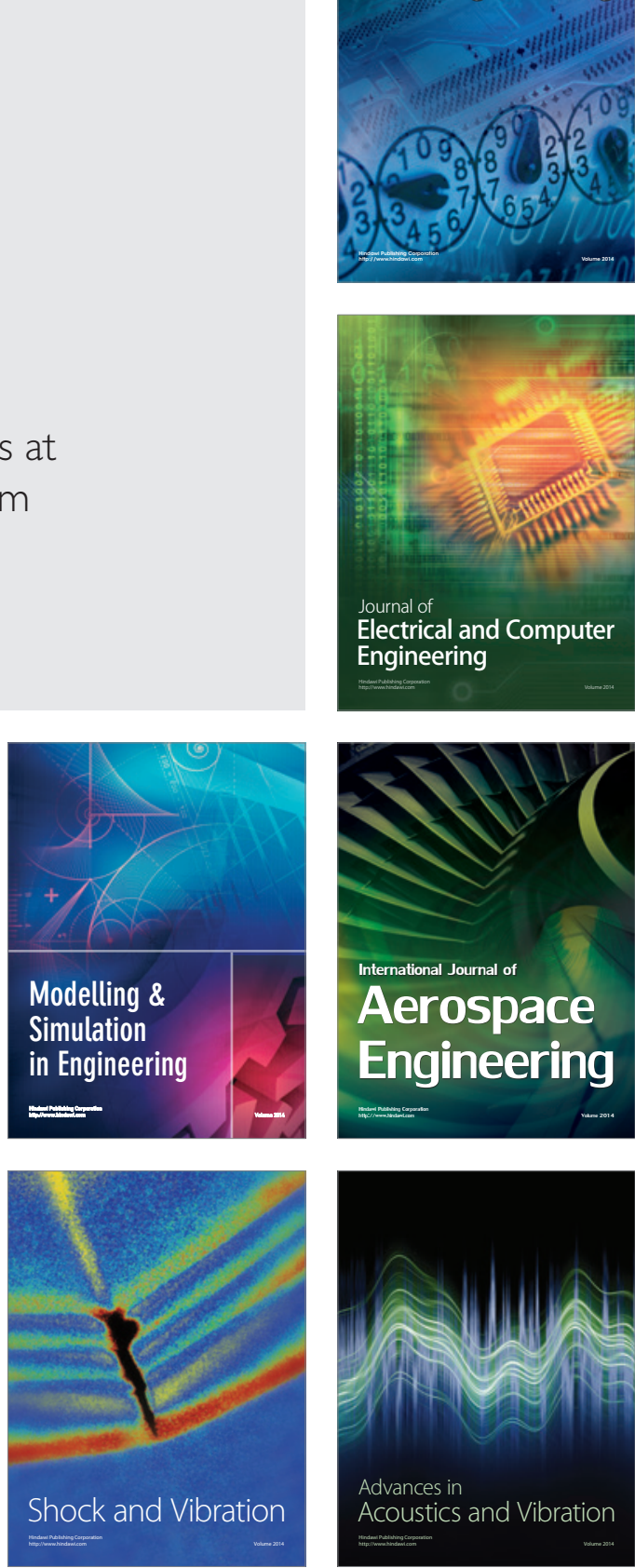